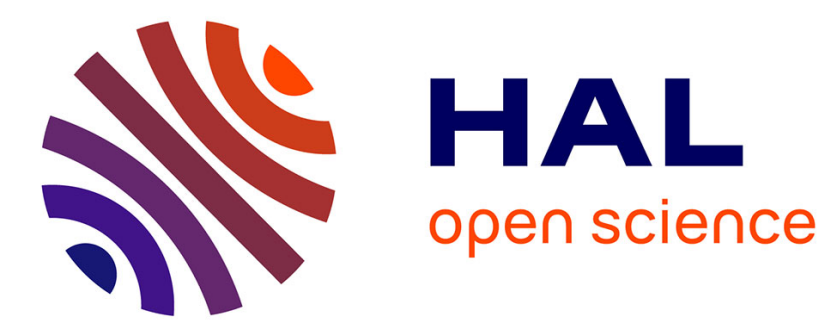

\title{
Dynamic Curing Agents for Amine-Hardened Epoxy Vitrimers with Short (Re)processing Times
}

Yann Spiesschaert, Marc Guerre, Ives de Baere, Wim van Paepegem, Johan Winne, Filip Du Prez

\section{- To cite this version:}

Yann Spiesschaert, Marc Guerre, Ives de Baere, Wim van Paepegem, Johan Winne, et al.. Dynamic Curing Agents for Amine-Hardened Epoxy Vitrimers with Short (Re)processing Times. Macromolecules, 2020, 53 (7), pp.2485-2495. 10.1021/acs.macromol.9b02526 . hal-02614115

\section{HAL Id: hal-02614115 https://hal.science/hal-02614115}

Submitted on 6 Jul 2021

HAL is a multi-disciplinary open access archive for the deposit and dissemination of scientific research documents, whether they are published or not. The documents may come from teaching and research institutions in France or abroad, or from public or private research centers.
L'archive ouverte pluridisciplinaire HAL, est destinée au dépôt et à la diffusion de documents scientifiques de niveau recherche, publiés ou non, émanant des établissements d'enseignement et de recherche français ou étrangers, des laboratoires publics ou privés. 


\section{Dynamic curing agents for amine-hardened epoxy vitrimers}

\section{with short processing times}

Yann Spiesschaert, ${ }^{1}$ Marc Guerre, ${ }^{1,3}$ Ives De Baere, ${ }^{2}$ Wim van Paepegem, ${ }^{2}$ Johan M. Winne, ${ }^{1 *}$ and Filip E. Du Prez ${ }^{1 *}$

${ }^{1}$ Polymer Chemistry Research group and Laboratory for Organic Synthesis, Department of Organic and Macromolecular Chemistry, Faculty of Sciences, Ghent University, Krijgslaan 281 S4bis, 9000 Ghent, Belgium

${ }^{2}$ Department of Materials, Textiles and Chemical Engineering, Ghent University, TechnologieparkZwijnaarde 46, 9052 Zwijnaarde, Belgium

${ }^{3}$ Laboratoire des IMRCP, Université de Toulouse, CNRS UMR5623, Université Paul Sabatier, 118 route de Narbonne, 31062 Toulouse Cedex 9

*Corresponding author: Filip.DuPrez@UGent.be and Johan.Winne@UGent.be

\section{Abstract}

This work presents a straightforward strategy to introduce highly dynamic and adaptable cross-links into common epoxy resin formulations. For this, an oligomeric amine-based curing agent containing vinylogous urethane (VU) bonds was developed. This novel polyfunctional amine curing agent can be used as a drop-in solution for existing epoxy resin technologies, resulting in transparent, rigid and, at the same time, highly reprocessable catalyst-free epoxy vitrimers. The oligomeric VU curing agents are prepolymerised via a straightforward condensation reaction between acetoacetates extended with different classical amine monomers and epoxy hardeners. It is found that vitrimer properties can be readily introduced into these epoxy formulations by converting less than 50 mol\% of the hardener's amine functionalities into dynamic vinylogous urethane bonds. In this way, epoxy vitrimers can be 
obtained with material properties comparable to ones of the VU-free epoxy formulations. In addition, remarkably short processing times are observed in the absence of any catalyst, and the material displayed very short stress relaxation times and good recyclability, actually representing the most performant VU-based vitrimers so far. Furthermore, a proof of concept for its use in obtaining glass fiber-reinforced epoxy composites is also presented.

Keywords: curing agent, epoxy resin, vinylogous urethane, vitrimer

\section{Introduction}

Epoxy polymers and monomers can be readily obtained from various sources, via oxidation of simple olefins or via alkylation of nucleophilic functionalities with, for example, epichlorohydrin. Thus, epoxy polymer technology is compatible with a very wide variety of material precursors, like epoxidized soybean oil, ${ }^{1}$ epoxidized polyisoprene ${ }^{2-4}$ and bisphenol A diglycidyl ether. ${ }^{5,6}$ Furthermore, epoxides are versatile bond-forming functionalities and can be reacted with a broad range of functional groups, e.g. thiols, ${ }^{7}$ amines, ${ }^{8,9}$ carboxylic acids ${ }^{10}$ and anhydrides. ${ }^{11}$ The strain-promoted ring-opening reactions of epoxides with various nucleophiles all have the advantage of not releasing small volatile compounds during the bond forming process. Reactivity can be fine-tuned for a given polymerization or curing process, also readily enabling the preparation of defect-free thermosetting epoxy networks, which is a major field of application for epoxy-based materials. The widespread and still growing interest in epoxy resins originates from their good chemical resistance, mechanical properties, electrical insulating behavior and excellent adhesion. Therefore, they can be found in a large span of applications ranging from coatings and adhesives, to high performance composite materials. ${ }^{5,6}$

Like all thermosets, current epoxy materials have a fixed molecular network structure and are thus unable to be repaired, reshaped or recycled, generating a significant amount of waste that is intrinsically non-recyclable once it is fully cured. Moreover, epoxy resins also necessarily have to be produced (synthesized) directly into their desired shape, or trimmed down to a final form, putting limitations on the material applications. To circumvent these problems, the scientific community has eagerly investigated the recently introduced concept of vitrimers. ${ }^{10}$ Vitrimers are a subfamily of 
covalent adaptable networks ${ }^{12}$ where a rapid exchange of crosslinks can be achieved, while the number of crosslinks remains constant, resulting in a permanent, structurally integer yet dynamic network that is insoluble, but at the same time also weldable, reprocessable and recyclable, mimicking a combination of properties more commonly found in inorganic vitreous glasses. ${ }^{13}$

For the design of vitrimers, a broad range of chemical platforms have been investigated in recent years, such as transesterification, ${ }^{10,14-20}$ transamination, ${ }^{21-30}$ disulfide exchange ${ }^{31-33}$, silyl ether ${ }^{34,35}$ and many more $^{36-42}$ The very first vitrimer material, developed by Leibler and coworkers, was in fact an epoxy resin-based formulation that can undergo catalysed transesterification. ${ }^{10}$ Other dynamic covalent chemistries have since then also been implemented in epoxy-based vitrimers, such as disulfide metathesis $^{32,33}$ and imine exchange..$^{28,30,43}$ These chemistries have enabled a completely new way of looking at classical epoxy materials. As with all scientific innovations, the take-up of the technology in epoxy materials is not always straightforward. Some of the factors contributing to this can be related to the fact that these chemistries may be chemically far removed from existing epoxy formulations, and for example require the incorporation of a catalyst ${ }^{10,17,18}$ throughout the bulk of the material, require specific non-commercial monomers ${ }^{28,} 30,44$, or hardeners with functionalities that are chemically and physicochemically quite different from those used in normal epoxy formulations.

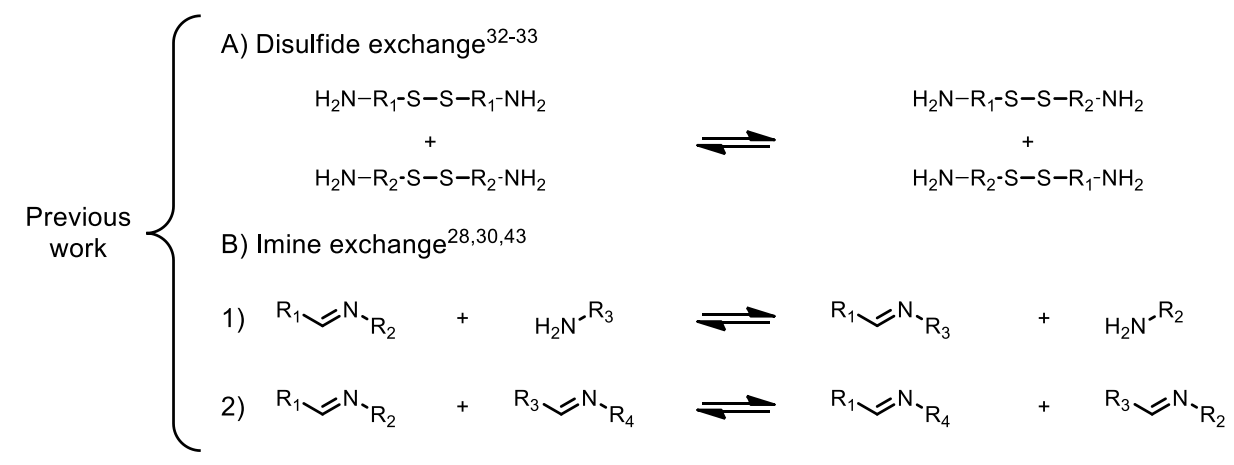

C) Vinylogous urethane exchange

This work
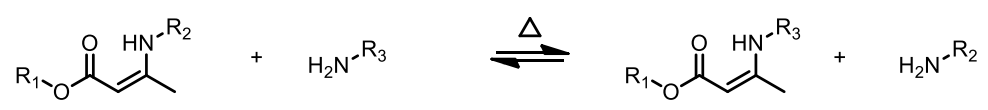

Figure 1. A\&B) Schematic overview of the two most applied dynamic exchange chemistries in amine cured epoxy vitrimers, A) disulfide exchange and B) imine exchange; C) Schematic overview of the dynamic exchange chemistry used in this work: vinylogous urethane exchange. 
In 2015 , we introduced vinylogous urethane chemistry for the design of vitrimers ${ }^{21}$. A drawback for that chemistry platform is that it relies on chemistries and monomers that, although certainly common in niche polymer materials, are not applied in high-volume bulk polymer materials. Therefore, at the outset of this study, we wanted to combine the vinylogous urethane platform with epoxy materials. So far, only few vitrimer chemistries have been applied in amine-hardened epoxy resins, with limited success $^{28,30,43,45}$ (Figure $1 \mathrm{~A}$ and B). For example, Odriozola et al. developed a highly interesting disulfide-based diamine epoxy hardener. ${ }^{32}$ These materials were later found to undergo dynamic bond exchanges mainly through a thermally initiated free radical addition-fragmentation type mechanism involving thiyl radicals adding into disulfide linkages, ${ }^{46}$ rather than a disulfide metathesis exchange. The chain-like mechanism of the bond exchange reactions leads to more complex thermal responses of the material compared to idealized vitrimers..$^{13,47}$

Herein, we report the use of the catalyst free vinylogous urethane (VU) chemistry platform (Figure 1C) for the preparation of a novel curing agent for epoxy vitrimers formulations. This study comprises the design and synthesis of the polyfunctional VU-based amine curing agent, the synthesis of aminehardened epoxy resins, and a study of their material properties in comparison to reference epoxy materials. In addition to a study of the rheological behavior enabled by the dynamic curing agent, also the recyclability of the obtained vitrimers is reported and a proof of concept for the utilization of vinylogous urethane epoxy vitrimers in glass-fiber reinforced composite materials is given. 


\section{Experimental section}

Materials

Benzyl alcohol (99+ \%) was purchased from Acros Organics. Zinc acetate dihydrate ( $\geq 99 \%)$ was purchased from Fluka. EPIKOTE ${ }^{\mathrm{TM}}$ Resin MGS LR 135 and EPIKURE ${ }^{\text {TM }}$ curing agent MGS LH 135 were kindly provided by Hexion. Ethylene glycol (99\%) was purchased from J\&K scientific. 1-Propanol (>99.5\%), 2,2,6-trimethyl-4H-1,3-dioxin-4-one $\quad(\geq 93 \%), 2$-hexanol $\quad(98 \%)$, benzylamine $\quad(99 \%)$, chloroform ( $\geq 99.8 \%$ ), tert-butyl acetoacetate ( $\geq 98 \%$ ) and tris(2-aminoethyl)amine (TREN, $96 \%$ ). 1,3-bis(aminomethyl)cyclohexane (98\%) were purchased from TCl Europe. Anhydrous dodecane (99+\%), 2-ethyl-1-hexylamine (98\%) and octylamine (99\%) were purchased from Sigma Aldrich. Glass-fiber fabric style 3025 was used from Porcher Industries. 2,2,6-Trimethyl-4H-1,3-dioxin-4-one was purified via distillation $\left(0.2 \mathrm{Torr}, 65-67^{\circ} \mathrm{C}\right)$. The other chemical products were used as received unless specified.

\section{Instrumentation}

Nuclear magnetic resonance (NMR) analyses were conducted on a Bruker Avance 300 (300 MHz) and a Brucker Avance II $400(400 \mathrm{MHz})$ to measure proton spectra at $25^{\circ} \mathrm{C}$. The NMR spectra were measured in $\mathrm{CDCl}_{3}$ and chemical shifts $(\delta)$ are presented in parts per million (ppm), relative to $\mathrm{CDCl}_{3}$ as the internal standard.

Thermogravimetric analyses (TGA) were performed on a Mettler-Toledo TGA/SDTA 851e instrument. All dynamic thermogravimetric measurements were performed under nitrogen atmosphere with a heating rate of $10 \mathrm{~K} / \mathrm{min}$ from $25^{\circ} \mathrm{C}$ to $800^{\circ} \mathrm{C}$. All isothermal thermogravimetric measurements were performed under nitrogen atmosphere at $150^{\circ} \mathrm{C}$ for 120 minutes with a heating rate of $10 \mathrm{~K} / \mathrm{min}$.

A Mettler Toledo instrument $1 / 700$ was used to perform differential scanning calorimetry (DSC) measurements under nitrogen atmosphere with a heating rate of $10^{\circ} \mathrm{C} / \mathrm{min}$ and a cooling rate of $10^{\circ} \mathrm{C} / \mathrm{min}$. Measurements were performed from $25^{\circ} \mathrm{C}$ to $150^{\circ} \mathrm{C}$. 
Stress-relaxation tests were performed using an Anton-Paar MCR 302 rheometer with a plate diameter of $8 \mathrm{~mm}$ and on samples having a diameter of $8 \mathrm{~mm}$ and a thickness of $1 \mathrm{~mm}$. Amplitude sweep experiments were performed using a frequency of $1 \mathrm{~Hz}$, a constant force of $5 \mathrm{~N}$ and a variable shear strain that was ramped up logarithmically from $0.01 \%$ to $100 \%$. Time sweep experiments with a constant shear strain of $1 \%$, a constant force of $5 \mathrm{~N}$ and an angular frequency of 1 rad. $\mathrm{s}^{-1}$ during which both the storage and the loss modulus were measured, were performed before every stress-relaxation measurement. Then stress-relaxation experiments were performed using a constant shear strain of $1 \%$ and a constant force of $5 \mathrm{~N}$. Creep experiments at different temperatures were also performed using a constant force of $5 \mathrm{~N}$. Additionally, in the first 300 s no shear stress was applied. Following this, a $5000 \mathrm{~Pa}$ shear stress was applied for $1200 \mathrm{~s}$ and finally the shear stress was removed again for another $1200 \mathrm{~s}$. Viscosity experiments were performed at a specified temperature and using a shear rate of $50 \mathrm{~s}^{-1}$.

Uniaxial tensile experiments were performed on a Tinius-Olsen H1OKT tensile tester, equipped with a $5000 \mathrm{~N}$ load cell and at a speed of $10 \mathrm{~mm} / \mathrm{min}$ and a pre-load of $0.05 \mathrm{~N}$. Flat dog-bone type specimens with an effective gauge length of $13 \mathrm{~mm}$, a width of $2 \mathrm{~mm}$ and a thickness of $1 \mathrm{~mm}$ were used for the tensile tests. The samples were cut out using a Ray-Ran dog-bone cutter, the samples and the dog-bone cutter were pre-heated for 5 minutes in a 7.5-ton manual rubberstamp press at $150{ }^{\circ} \mathrm{C}$. The tensile tests performed on the composite samples were carried out on a servo-hydraulic INSTRON 8801 tensile testing machine utilizing a load cell of $\pm 100 \mathrm{kN}$, a FastTrack 8800 digital controller and a speed of $2 \mathrm{~mm} / \mathrm{min}$. In order to prevent relative rotation of the grips, a special fixture is mounted on the tensile machine. Additionally, an INSTRON alignment kit was used to align the grips. The tensile data was registered using a National Instruments C-series data acquisition card. The signals from strain gauges as well as the load, displacement and strain recorded by the FastTrack controller were measured on the same time basis. The samples had a rectangular shape of $3 \times 20 \mathrm{~cm}^{2}$. 
Attenuated total reflection Fourier transform Infrared (ATR-FTIR) analyses were realized on a Perkin-Elmer Spectrum1000 FTIR infrared spectrometer equipped with a diamond ATR probe.

A SpeedMixer ${ }^{\mathrm{TM}}$ DAC $150.1 \mathrm{FVZ}$ was used to homogenize the samples.

The networks were (re)processed using compression molding. The samples were cut into small pieces and placed in the mold. They were then put in a heated press at $150{ }^{\circ} \mathrm{C}$ for 15 minutes at 0 metric ton pressure, after which the pressure was increased to 0.25 metric tons for 20 minutes. Following this, the pressure was further raised to 1 metric tons for a final 20 minutes. The samples were then removed from the mold while they were still warm.

Soxhlet experiments were performed using toluene at $125^{\circ} \mathrm{C}$ for $24 \mathrm{~h}$. Then, the samples were carefully taken out and were dried in a vacuum oven at $110^{\circ} \mathrm{C}$ overnight to remove the remainder of the solvent. The soluble fraction was calculated using Equation (1). The reported results are averages of three measurements.

$$
\text { Soluble fraction }(\%)=\frac{m_{\text {initial }}-m_{d r y}}{m_{\text {initial }}} \times 100 \%
$$

\section{Synthetic procedures}

Propyl acetoacetate was prepared following a procedure described by Denissen et al. ${ }^{21}$ Briefly, 2,2,6trimethyl-4H-1,3-dioxin-4-one (1 eq.) and 1-propanol (4 eq.) were mixed in a pressure tube and heated for $3 \mathrm{~h}$ at $135^{\circ} \mathrm{C}$. After the reaction was finished according to thin layer chromatography (TLC), the excess of 1-propanol was removed under vacuum, yielding pure propyl acetoacetate (Yield $=98 \%) .{ }^{1} \mathrm{H}$ $\operatorname{NMR}\left(300 \mathrm{MHz}, \mathrm{CDCl}_{3}, \delta\right): 4.10(\mathrm{t}, \mathrm{J}=6.5 \mathrm{~Hz}, 2 \mathrm{H}), 3.45(\mathrm{~s}, 2 \mathrm{H}), 2.27(\mathrm{~s}, 3 \mathrm{H}), 1.63(\mathrm{~m}, 2 \mathrm{H}), 0.94(\mathrm{t}, \mathrm{J}=6.5$ $\mathrm{Hz}, 3 \mathrm{H})$.

Propyl-3-(butylamino)but-2-enoate was synthesized following a protocol proposed by Denissen et al. ${ }^{21}$ Propyl acetoacetate (1 eq.) and butylamine ( 2 eq.) were dissolved in $5 \mathrm{ml}$ of methanol and the mixture was stirred overnight. When the formation of the vinylogous urethane was complete (followed via thin 
layer chromatography (TLC)), the solvent was removed under vacuum. The mixture is then dissolved in dichloromethane and washed twice with brine. The organic phase was dried with $\mathrm{MgSO}_{4}$ and the solvent evaporated yielding the desired product. The obtained product was further purified by flash chromatography using EtOAc/hexane (25/75). Yield $=92 \%{ }^{1} \mathrm{H}$ NMR $\left(300 \mathrm{MHz}, \mathrm{CDCl}_{3}, \delta\right): 8.55(\mathrm{~s}, 1 \mathrm{H})$, $4.44(\mathrm{~s}, 1 \mathrm{H}), 3.98(\mathrm{t}, \mathrm{J}=6.77 \mathrm{~Hz}, 2 \mathrm{H}), 3.20(\mathrm{q}, \mathrm{J}=6.52 \mathrm{~Hz}, 2 \mathrm{H}), 1.91(\mathrm{~s}, 3 \mathrm{H}), 1.65-1.39(\mathrm{~m}, 7 \mathrm{H}), 0.936(\mathrm{t}$, $\mathrm{J}=7.37 \mathrm{~Hz}, 6 \mathrm{H})$.

Synthesis of propyl-3-(octylamino)but-2-enoate. Octyl amine (1.5 eq., $26 \mathrm{mmol}$ ) was added to propyl acetoacetate $\left(1\right.$ eq., $17 \mathrm{mmol}$ ) in chloroform $(30 \mathrm{ml})$ and $\mathrm{NaSO}_{4}(1 \mathrm{~g})$. The mixture was left to stir overnight at room temperature. Afterwards it was filtered over silica to remove the excess of amines. Yield $=91 \% .{ }^{1} \mathrm{H}$ NMR $\left(300 \mathrm{MHz}, \mathrm{CDCl}_{3}, \delta\right): 8.53(\mathrm{~s}, 1 \mathrm{H}), 4.42(\mathrm{~m}, 1 \mathrm{H}), 3.97(\mathrm{~m}, 2 \mathrm{H}), 3.17(\mathrm{~m}, 2 \mathrm{H}), 1.89$ (s, 3H), 1.68-1.48 (m, 4H), 1.4-1.2 (m, 10H), 0.94-0.83 (m, 6H).

Transesterification of propyl-3-(butylamino)but-2-enoate was realized with 3 different protocols:

1) Propyl-3-(butylamino)but-2-enoate was mixed with either benzyl alcohol or 2-hexanol (2 eq.). Following this, for the reactions containing catalyst, $5 \mathrm{~mol} \%$ of $\mathrm{Zn}$ (acetate). $2 \mathrm{H}_{2} \mathrm{O}$ was also added. The mixtures were heated up to $100{ }^{\circ} \mathrm{C}$ (for benzyl alcohol) or $120^{\circ} \mathrm{C}$ (for 2-hexanol) in bulk and at specific time intervals, NMR samples were taken.

2) Propyl-3-(butylamino)but-2-enoate was mixed with 2-hexanol (2 eq.) and heated to $160{ }^{\circ} \mathrm{C}$ in a pressure tube in bulk. At specific time intervals, NMR samples were taken.

3) Propyl-3-(butylamino)but-2-enoate was mixed with 2-hexanol (2 eq.) and di-isopropylethylamine (0.5 eq.) and heated to $120^{\circ} \mathrm{C}$ in bulk. At specific time intervals, NMR samples were taken.

Transamination of propyl-3-(octylamino)but-2-enoate. Propyl-3-(octylamino)but-2-enoate (1 eq.) was mixed with benzylamine ( 5 eq.) and 2-hexanol ( 2 eq.) or dodecane ( 2 eq.) in bulk. The mixture was then heated to $100{ }^{\circ} \mathrm{C}$ and NMR samples were taken at specific time intervals. 
Synthesis of ethylene glycol-bisacetoacetate (EG-AA). Ethylene glycol (1 eq.) was mixed together with tert-butyl acetoacetate ( 2.3 eq.) in a distillation set-up. The mixture was then heated to $125^{\circ} \mathrm{C}$ at $800 \mathrm{mbar}$ for $3 \mathrm{~h}$ to remove the formed tert-butanol. Afterwards, the pressure was reduced gradually to $5 \mathrm{mbar}$ in the timespan of $1 \mathrm{~h}$. Finally, to remove the final traces of tert-butanol and tertbutylacetoacetate, the mixture was left for another $1 \mathrm{~h}$ at $5 \mathrm{mbar}$. No further purification was performed. Yield $=95 \% .{ }^{1} \mathrm{H}$ NMR $\left(400 \mathrm{MHz}, \mathrm{CDCl}_{3}, \delta\right): 11.87(\mathrm{~s}, 2 \mathrm{H}$, enol), $4.99(\mathrm{~s}, 2 \mathrm{H}$, enol), 4.35 (s, $4 \mathrm{H}), 3.47(\mathrm{~s}, 4 \mathrm{H}), 2.25(\mathrm{~s}, 6 \mathrm{H}), 1.95(\mathrm{~s}, 6 \mathrm{H}$, enol).

Vinylogous urethane curing agent (VU curing agent) preparation. EG-AA (1 eq.) was added to a solution of 1,3-bis(aminomethyl)cyclohexane (BAC) (1.75 eq.) and tris(2-aminoethyl)amine (0.5 eq.). The mixture was then mixed using a speedmixer for $2 \times 2$ minutes at $2500 \mathrm{rpm}$. Following this, the mixture was dried in a vacuum oven at $65^{\circ} \mathrm{C}$ to remove the formed water.

Synthesis of the reference epoxy network 1 (ER 1): EPIKOTE ${ }^{\mathrm{TM}}$ resin MGS LR 135 and EPIKURE ${ }^{\mathrm{TM}}$ curing agent MGS LH 135 were mixed in a ratio of 100:35 \pm 2 (LR135:LH135) as provided by Hexion. The mixture was mixed using a speedmixer for 2 minutes at $2500 \mathrm{rpm}$, after which it was left at $45^{\circ} \mathrm{C}$ for $1 \mathrm{~h}$ and then fully cured at $100^{\circ} \mathrm{C}$ in a vacuum oven overnight.

Network synthesis using water-free network (WFN) procedure. The pre-polymer was mixed with EPIKOTE ${ }^{\mathrm{TM}}$ resin MGS LR 135 using a speedmixer for $2 \times 2$ minutes at $2500 \mathrm{rpm}$ in an adapted ratio from 100:35 \pm 2 (LR135:LH135) to ensure a 5\% excess of primary amines. Then it was cured for $1 \mathrm{~h}$ at $90{ }^{\circ} \mathrm{C}$ and afterwards transferred to a vacuum oven at $100{ }^{\circ} \mathrm{C}$ overnight.

Network synthesis using one-pot network (OPN) procedure. EG-AA (1 eq.) was mixed with BAC (1.75 eq.), TREN (0.5 eq.) and EPIKOTE TM resin MGS LR 135 using a speedmixer for $2 \times 2$ minutes at $2500 \mathrm{rpm}$ in an adapted ratio from 100:35 \pm 2 (LR135:LH135) to ensure a 5\% excess of primary amines. Then it was cured for $1 \mathrm{~h}$ at $90^{\circ} \mathrm{C}$ and afterwards transferred to a vacuum oven at $100{ }^{\circ} \mathrm{C}$ overnight. 
Synthesis of the reference epoxy network 2 (ER 2): EPIKOTE ${ }^{\text {TM }}$ resin MGS LR 135 was mixed with BAC and TREN (3.5:1) in an adapted ratio of 100:35 \pm 2 (LR135:LH135) to ensure a 10\% excess of primary amines. The mixture was mixed using a speedmixer for 2 minutes at $2500 \mathrm{rpm}$, after which it was left at $45^{\circ} \mathrm{C}$ for $1 \mathrm{~h}$ and then fully cured at $100{ }^{\circ} \mathrm{C}$ in a vacuum oven overnight.

Synthesis of the reference VUR network: EG-AA was mixed together with BAC and TREN (3.5:1) using a speedmixer for $2 \times 2 \mathrm{~min}$ at $2500 \mathrm{rpm}$. Then it was cured for $1 \mathrm{~h}$ at $90^{\circ} \mathrm{C}$ and afterwards transferred to a vacuum oven at $100^{\circ} \mathrm{C}$ overnight.

Composite formation. One layer of glass fiber was placed on a none-sticky foil. EPIKOTE ${ }^{\mathrm{TM}}$ resin MGS LR 135 and the VU curing agent were separately heated to $45^{\circ} \mathrm{C}$. Once both were at $45^{\circ} \mathrm{C}$, they were mixed together and partially poured on the glass fiber. The poured epoxy mixture was then manually spread using a pressure roller until all fibers were impregnated. Following this, a next layer of glass fiber was placed on top of the impregnated layer of glass fiber. A next amount of epoxy resin was poured on the new layer of glass fiber and again manually spread. This process was repeated until the desired layers of glass fiber were reached. Then the foil was closed in a bag and sealed under vacuum. The composite was then placed in an oven at $100{ }^{\circ} \mathrm{C}$ and cured for $4 \mathrm{~h}$.

Composite thermoforming. The sample was placed in a pre-heated oven at $150{ }^{\circ} \mathrm{C}$ for 10 minutes together with the weights used for reshaping. Once the sample and the weights were at the right temperature, the sample was gradually deformed. After the desired deformation was reached, the sample was kept in its new shape for 15 minutes to ensure full stress release due to reorganization of the vinylogous urethane matrix. Following this, the weights were removed, and the sample was left to cool down. The sample maintained its new shape without visible damage to the matrix. 


\section{Results and Discussion}

Investigation of a curing agent for water-free preparation of vinylogous urethane vitrimers

In previous studies, ${ }^{21,24,29,48}$ the synthesis of the vinylogous urethanes and the network formation (VU networks) occurred simultaneously via the reaction between a bi- or tri-functional amine with a bi- or tri-functional acetoacetate. However, this procedure resulted in the release of water during network formation, which lead to material defects and thus the necessity for a reprocessing step to prepare homogeneous materials. In other words, the starting point of this study was the development of defect-free VU networks, using crosslinking approaches based on the well-known amine epoxy reaction that does not release any small volatile compounds. In this context, two methods to obtain vinylogous urethane-containing epoxy vitrimers using a mixture of tris(2-aminoethyl)amine (TREN), 1,3-bis(aminomethyl)cyclohexane (BAC), ethylene glycol bis-acetoacetate (EG-AA) and an industrially applied epoxy mixture (EPIKOTE TM Resin MGS LR 135) have been compared (Figure 2).

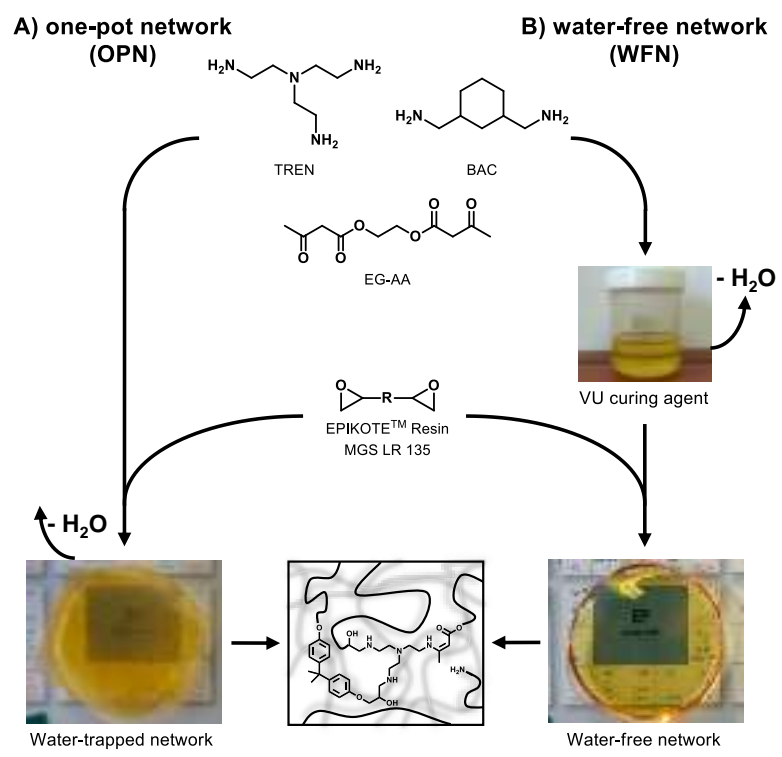

Figure 2. Schematic overview of the different strategies utilized to obtain vinylogous urethane epoxy materials. A) one-pot network method (OPN) with water release and B) water-free network method (WFN) using a vinylogous urethane-based curing agent. 
In the first method, in which all compounds are reacted simultaneously, further referred to as the one-pot network procedure (OPN, Figure 2A), water is released during the network formation resulting in water bubbles trapped inside the vitrimer, thus requiring an additional processing step (grinding/compression molding) to obtain bubble free networks. In the second method, the so-called water-free network procedure (WFN, Figure 2B), a VU-based curing agent is first prepared, dried and used as a regular commercial curing agent for epoxy materials. Since the water is already removed, during the preparation of the curing agent, bubble formation is prevented during the curing step. Therefore, the material can be directly used and cured in its final shape similarly to conventional, unrecyclable epoxy thermosets. In this procedure, the VU curing agents fulfills three key requirements: its primary amine groups are necessary to cure the epoxy resin and to promote the VU exchange reactions, and it has a relatively low viscosity for processability. Indeed, low viscosity epoxy precursors show several benefits such as good adhesion and wettability of the resins. Moreover, they allow for their use in a variety of processing techniques such as resin transfer molding ${ }^{49}$ or vacuum infusion, ${ }^{50}$ which are used to obtain composite materials such as wind turbine blades. In order to achieve this low viscosity, the amount of branching needs to be kept low. In this context, a ratio of 3.5:1 of BAC:TREN (Fig. 2) was chosen to be investigated for its use as a potential VU curing agent.

In order to obtain materials with similar properties as standard epoxy resins, the amount of reversible linkages must be kept reasonably low. Nonetheless, a correct balance between property requirements and processability conditions needs to be met. In a recent study reported by Torkelson and co-workers, a theoretical formula is presented to calculate the upper limit of permanent crosslinks that can be present before the material loses its processability. ${ }^{51}$ This formula (Equation S1) was experimentally validated using a highly crosslinked system, where it was found that, for the investigated composition, a maximum of $40 \%$ permanent crosslinks could be present to maintain recyclability. That same formula was applied to the here described system, with the assumption that only primary amines can react with epoxides, and it was theoretically found that at least $30 \%$ dynamic bonds (and thus a maximum of $70 \%$ permanent bonds) should be present to provide reprocessability (Table S1). To experimentally 
validate this value, different materials were synthesized via the above-described classical OPN method with varying amounts of dynamic vinylogous urethane bonds, ranging from $20 \mathrm{~mol} \%$ to $60 \mathrm{~mol} \%$. These tests displayed that good processability could be obtained when using concentrations of vinylogous urethane bonds above 40 mol\%, while the material made with 30 mol\% vinylogous urethane bonds showed only partial processability (Figure S1). This difference between theoretical and experimental values could most likely be ascribed to the assumption that only primary amines react and the resulting underestimation of possible irreversible links. Therefore, the composition containing $40 \mathrm{~mol} \%$ vinylogous urethane bonds and at least $5 \%$ excess of amines (necessary for the transamination reaction ${ }^{21}$ ) was chosen for an in-depth characterization. This composition both ensures the processability of the vitrimer and comparability with the epoxy reference material.

\section{Material characterization}

The aforementioned composition was utilized to obtain vinylogous urethane epoxy vitrimers using both the OPN and WFN approaches (OPN $\mathrm{O}_{40 / 5}$ and $\mathrm{WFN}_{40 / 5}$, respectively). Additionally, several reference samples were synthesized as benchmark. One reference sample is a commercially available epoxy network (ER 1) obtained via the mixing of EPIKOTE ${ }^{\mathrm{TM}}$ Resin MGS LR 135 and EPIKURE ${ }^{\mathrm{TM}}$ curing agent MGS LH 135. The second reference sample (ER 2) consists of an epoxy network containing $10 \%$ excess of amines, which is obtained via the mixing of EPIKOTE ${ }^{\mathrm{TM}}$ Resin MGS LR 135 with TREN and BAC. The final reference sample is an epoxy-free vinylogous urethane vitrimer (VUR).

Table 1. Number of recycling steps, thermal and tensile properties, as well as soluble fractions of all the obtained materials.

\begin{tabular}{|c|c|c|c|c|c|c|}
\hline Networks & $\begin{array}{c}\text { Recycling } \\
\text { steps }\end{array}$ & $\begin{array}{l}\mathrm{T}_{\mathrm{g}}^{\mathrm{a}} \\
\left({ }^{\circ} \mathrm{C}\right)\end{array}$ & $\begin{array}{l}\mathrm{T}_{\mathrm{d} 5 \%}{ }^{\mathrm{b}} \\
\left({ }^{\circ} \mathrm{C}\right)\end{array}$ & $\begin{array}{l}\text { Soluble } \\
\text { fraction } \\
\text { (\%) }\end{array}$ & $\begin{array}{l}\varepsilon^{d} \\
(\%)\end{array}$ & $\begin{array}{c}E^{\prime d} \\
(M P a)\end{array}$ \\
\hline WFN $_{40 / 5}$ & $\mathrm{RxO}$ & 74 & 248 & 1.4 & $13.50 \pm 1.19$ & $1560 \pm 50$ \\
\hline
\end{tabular}




\begin{tabular}{|c|c|c|c|c|c|c|}
\hline & $\mathrm{R} \times 1$ & 74 & $*$ & $*$ & $4.85 \pm 0.68$ & $1500 \pm 7$ \\
\hline & $\mathrm{R} \times 2$ & 75 & * & $*$ & $4.31 \pm 1.37$ & $1520 \pm 82$ \\
\hline & $\mathrm{R} \times 3$ & 74 & $*$ & * & $2.79 \pm 0.61$ & $1530 \pm 66$ \\
\hline & $\mathrm{R} \times 4$ & 75 & 252 & 0.7 & $2.80 \pm 0.14$ & $1560 \pm 54$ \\
\hline & $\mathrm{R} \times 0$ & 74 & 250 & 5.0 & $10.60 \pm 1.98$ & $1680 \pm 25$ \\
\hline & $\mathrm{R} \times 1$ & 74 & $*$ & $*$ & $3.92 \pm 1.65$ & $1710 \pm 101$ \\
\hline $\mathrm{OPN}_{40 / 5}$ & $\mathrm{R} \times 2$ & 73 & $*$ & $*$ & $3.66 \pm 0.81$ & $1620 \pm 36$ \\
\hline & $\mathrm{R} \times 3$ & 73 & $*$ & * & $4.05 \pm 1.03$ & $1650 \pm 36$ \\
\hline & $\mathrm{Rx} 4$ & 74 & 247 & 1.0 & $4.67 \pm 1.34$ & $1610 \pm 45$ \\
\hline ER 1 & $\mathrm{RxO}$ & 82 & 313 & 3.0 & $7.44 \pm 0.71$ & $1430 \pm 60$ \\
\hline ER 2 & $\mathrm{RxO}$ & 75 & 333 & 0.6 & $2.08 \pm 0.63$ & $1540 \pm 152$ \\
\hline VUR & $\mathrm{R} \times 0$ & 73 & 254 & 16.0 & $4.18 \pm 1.47$ & $1600 \pm 87$ \\
\hline
\end{tabular}

a Glass transition $\left(\mathrm{T}_{\mathrm{g}}\right)$ determined during second heating by differential scanning calorimetry (DSC) with a heating and cooling rate of $10 \mathrm{~K} / \mathrm{min}$; ${ }^{b}$ Temperature at which a weight loss of $5 \%$ is observed by thermogravimetric analysis (TGA); ${ }^{\mathrm{c}}$ Determined via Soxhlet extraction in refluxing toluene for $24 \mathrm{~h}$ at $135^{\circ} \mathrm{C}$; ${ }^{d}$ Apparent Young's modulus ( $\left.E^{\prime}\right)$ and elongation at break $(\varepsilon)$ determined from tensile testing $(0.05 \mathrm{~N}$ preload and $10 \mathrm{~mm} / \mathrm{min})$, strain is measured as [actuator displacement]/[gauge length]. These values are relative and used for comparison purposes only. * Not performed.

First, the thermal properties of these materials were investigated by DSC and TGA and the results are summarized in Table 1. The obtained values for the glass transition temperatures of the vitrimers and the references are all very similar and situated around $73-75^{\circ} \mathrm{C}$. Only a slight difference is noticeable between ER 1 and the other samples, which can be attributed to the fact that ER 1 is obtained following an optimized commercial formulation. This result is promising, since it proves the possibility to obtain epoxy vitrimers with potential recyclability while having a similar $\mathrm{T}_{\mathrm{g}}$ range as the commercially available counterparts. On the other hand, as expected, a larger difference was observed when comparing the thermal stability of the networks. The materials containing vinylogous urethane bonds have a $T_{d 5 \%}$ of around $250^{\circ} \mathrm{C}$, which is much lower compared to the one obtained for the epoxy references 
(comprised between 313 and $333^{\circ} \mathrm{C}$ ) (Figure $3 \mathrm{~A}$ ). These results are attributed to the degradation of the vinylogous urethane bonds, as already reported in the literature. ${ }^{21,48}$ Isothermal TGA analyses at $150{ }^{\circ} \mathrm{C}$ (typical reprocessing temperature) for 120 minutes displayed no significant difference with only a weight loss up to $1 \%$ after 120 minutes (Figure S2).
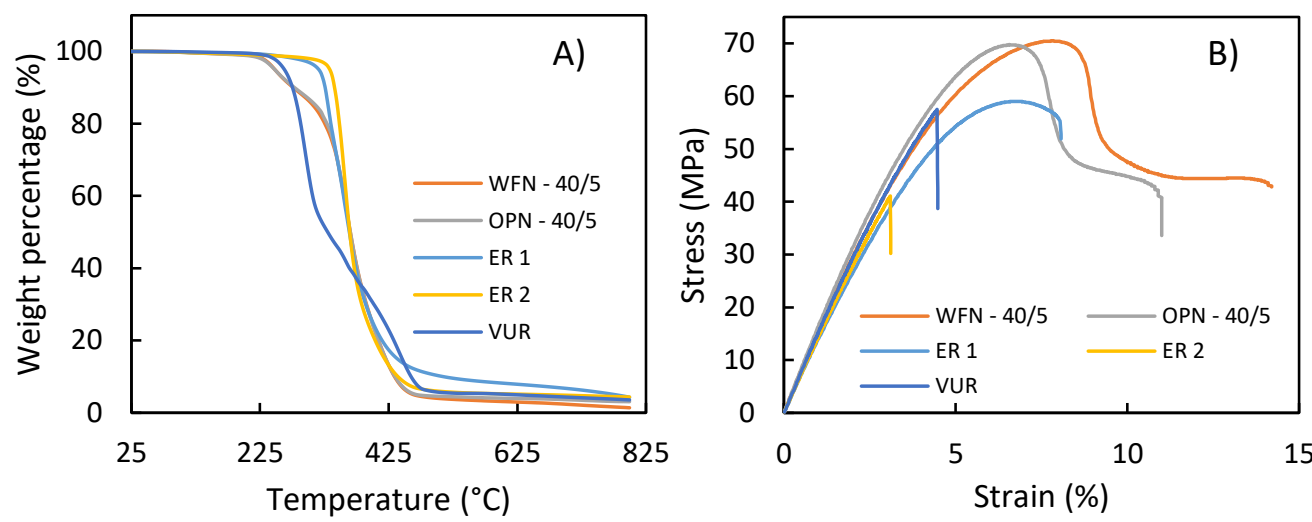

Figure 3. A) TGA of the different materials with a temperature ramp from 25 to $800{ }^{\circ} \mathrm{C}$ at $10{ }^{\circ} \mathrm{C} / \mathrm{min}$. B) Stress-strain curves of the different materials.

Secondly, the network integrity of all materials was investigated via the determination of the soluble fraction using Soxhlet extraction. The soluble fractions calculated for the two synthesized epoxy vitrimers $\left(\mathrm{OPN}_{40 / 5}\right.$ and $\left.\mathrm{WFN}_{40 / 5}\right)$ were lower than or equal to $5 \%$ after $24 \mathrm{~h}$ in toluene at $135^{\circ} \mathrm{C}$ indicating good network integrity.

Lastly, the tensile properties of $\mathrm{WFN}_{40 / 5}$ and $\mathrm{OPN}_{40 / 5}$ were compared to the different reference materials (Figure 3B). The materials displayed very similar apparent Young's moduli (Table 1). However, a slightly higher $E^{\prime}$ value was obtained for the $\mathrm{OPN}_{40 / 5}$ network (E' close to $1.7 \mathrm{GPa}$ ), which tends to indicate a beneficial interest of combining a vinylogous urethane matrix with an epoxy matrix for the OPN method. This result can be attributed to the mixing methods that can result in slightly different network structures. Indeed, a potentially more homogenous matrix should be obtained with the OPN method as a result of the lower viscosity that leads to a better mixing, and to the simultaneous substitution/condensation reactions. Furthermore, when comparing the tensile graphs (Figure 3B), a clear difference is visible between the references and the materials obtained with OPN and WFN. The 
references displayed a brittle fracture with an elongation between $4-7 \%$, while $\mathrm{OPN}_{40 / 5}$ and $\mathrm{WFN}_{40 / 5}$ displayed some necking, resulting in a much higher elongation (> $10 \%)$. These results indicate that the newly prepared vinylogous urethane epoxy vitrimers display similar or even better properties as the investigated reference materials.

\section{Viscoelastic behavior characterization}

Considering the large proportion of irreversible bonds in the matrix (60 mol\%), the viscoelastic properties and thus potential recyclability of $\mathrm{WFN}_{40 / 5}$ and $\mathrm{OPN}_{40 / 5}$ were verified by stress-relaxation experiments. As visible in Figure 4, both materials exhibit full stress-relaxation following a single exponential decay relaxation, which reflects a typical Maxwell behavior.
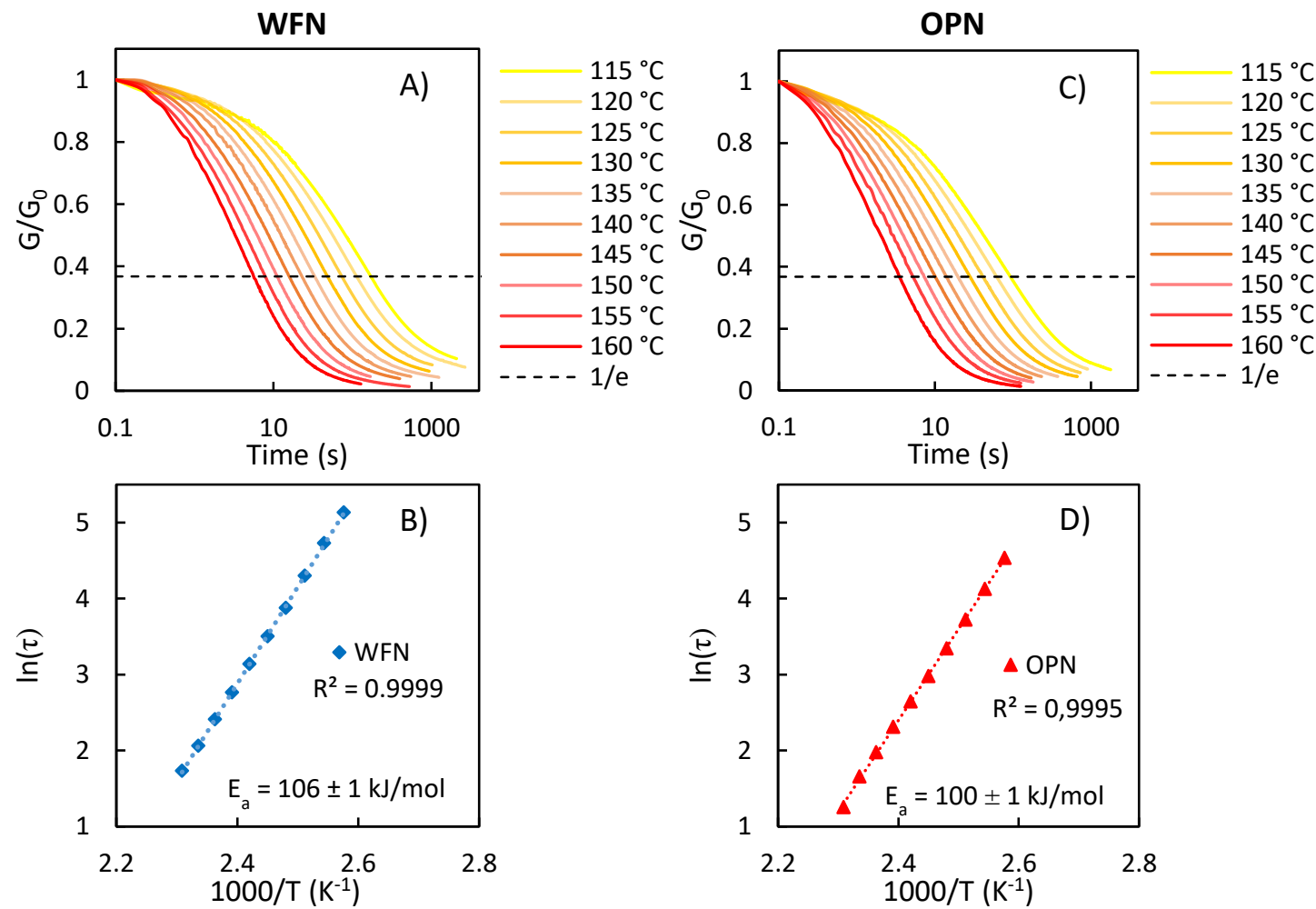

Figure 4. $A$ and C) Stress relaxation experiments of $W F N_{40 / 5}(A)$ and $O P N_{40 / 5}(C)$ from $115-160{ }^{\circ} \mathrm{C}$. The dashed line corresponds to the value 1/e, which defines the characteristic relaxation time $\tau$. A constant strain of $1 \%$ was applied, which was within the linear viscoelastic region. $B$ and D) Arrhenius plot of the obtained relaxation times of WFN $40 / 5(B)$ and $O P N_{40 / 5}$ (D) with a linear trend line. 
Figure $4 \mathrm{~A}$ and $\mathrm{C}$ show a nearly identical stress-relaxation behavior of $\mathrm{WFN}_{40 / 5}$ and $O P \mathrm{~N}_{40 / 5}$. The relaxation times measured for these two samples are also relatively close to each other with low values of $5.5 \mathrm{~s}$ and $3.5 \mathrm{~s}$ at $160{ }^{\circ} \mathrm{C}$ for $\mathrm{WFN}_{40 / 5}$ and $\mathrm{OPN}_{40 / 5}$, respectively. Furthermore, in both cases a near perfectly linear behavior is obtained in the Arrhenius plots (Figure $4 \mathrm{~B}$ and $\mathrm{D}$ ), confirming the vitrimernature of these materials despite the high proportion of irreversible bonds. Additionally, the activation energies $\left(E_{a}\right)$ obtained for these two samples are similar, namely $106 \pm 1 \mathrm{~kJ} / \mathrm{mol}$ and $100 \pm 1 \mathrm{~kJ} / \mathrm{mol}$ for $\mathrm{WFN}_{40 / 5}$ and $\mathrm{OPN}_{40 / 5}$, respectively. These activation energies are in the range of reported values for vinylogous urethane vitrimers. ${ }^{21,29,48}$

The obtained results were then compared to VUR, where a much slower exchange rate was observed with a relaxation time of $25.5 \mathrm{~s}$ at $160^{\circ} \mathrm{C}$. This was initially unexpected considering the higher concentration of exchangeable bonds in VUR compared to WFN $\mathrm{W}_{40 / 5}$ and $O P \mathrm{~N}_{40 / 5}$. Nevertheless, based on this significant difference in relaxation time, it is hypothesized that the addition of epoxides, and thus the resulting formation of secondary hydroxyl groups within a VU-matrix (Figure 5A), increases the transamination rate of vinylogous urethanes. Indeed, it was already demonstrated that VU exchange is accelerated in a protic environment, for example in the presence of para-toluene sulfonic acid. ${ }^{23}$ In addition, the chemical environment can have an influence on the exchange mechanism by shifting it towards either the Michael addition (Figure 5B) or the iminium (Figure S5) pathway. ${ }^{48}$ 
A)

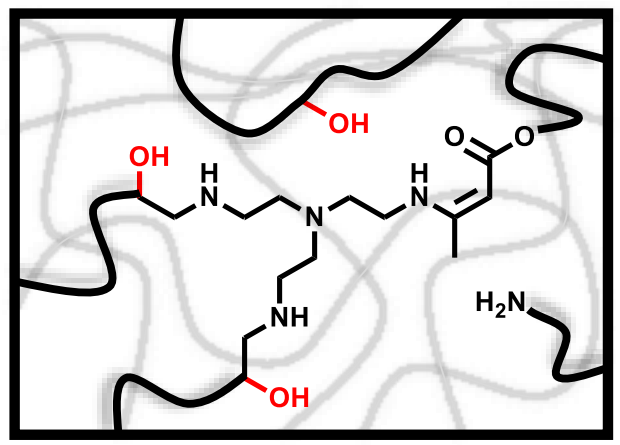

B)

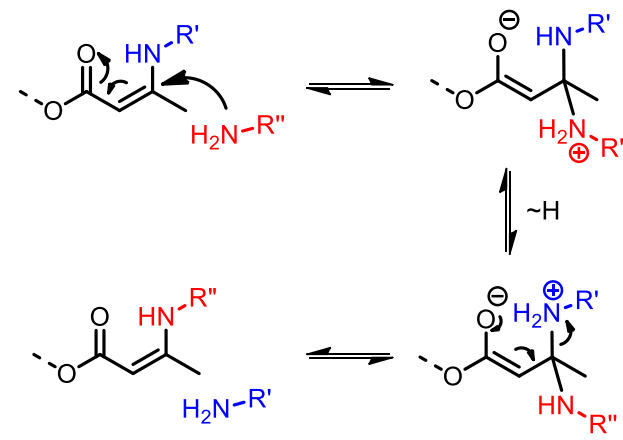

C) 1<smiles>CCCN/C(C)=C\C(=O)OCCC</smiles>
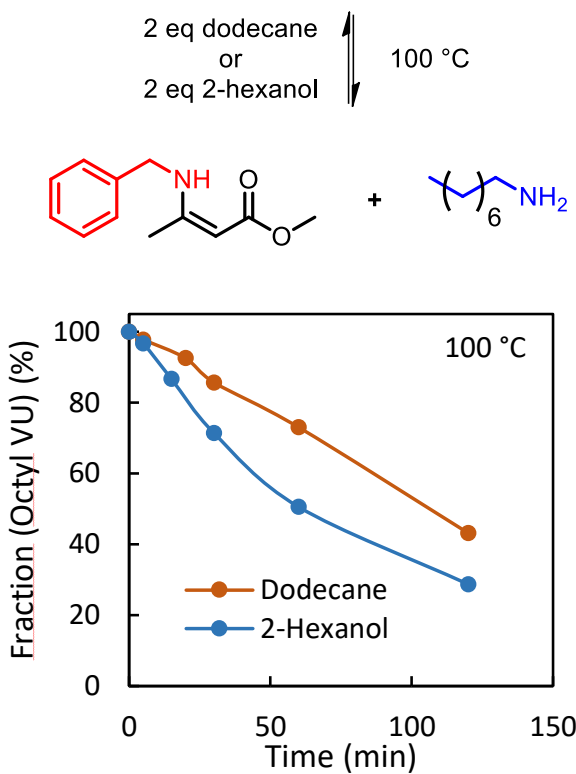

Figure 5. A) Schematic representation of the vitrimer network obtained via OPN or WFN, including the presence of hydroxyl groups. B) Exchange mechanism following a Michael-type pathway $E_{a}^{r e f}=102-122 \mathrm{~kJ} / \mathrm{mol}$. C) Model study in which the disappearance of propyl-3-(octylamino)but-2-enoate (octyl VU) is followed as a function of time, through exchange reactions with benzyl amine in the presence of dodecane or 2-hexanol at $100{ }^{\circ} \mathrm{C}$.

With the aim to evaluate the influence of hydroxyl groups on the vinylogous urethane transamination exchange kinetics, two model studies with small vinylogous urethane compounds were performed (Figure 5C). The transamination of propyl-3-(octylamino)but-2-enoate (octyl VU) with benzylamine was followed as a function of time in the presence of either 2 -hexanol or dodecane at $100{ }^{\circ} \mathrm{C}$. 2Hexanol was chosen for its high boiling point and for its secondary hydroxyl group that mimics the hydroxyl-containing epoxy vitrimers, while dodecane served as a non-hydroxyl containing reference to remove concentration effects. As shown in Figure 5C, which represents the fraction of octyl VU as a function of time, the reaction in 2-hexanol immediately resulted in a faster exchange (lower octyl VU fraction) compared to the reference experiment with dodecane. Based on these model reactions, it can be rationalized that the hydroxyl groups, resulting from the opening of epoxides, can indeed be 
responsible for the observed accelerating effect. This confirms our earlier statement ${ }^{13,52}$ that, not only the nature of the exchange chemistry and the catalyst are important when designing new vitrimer materials, but that also the effect of the matrix on the exchange reaction should be taken into account (e.g. internal catalysis effect).

Another important viscoelastic property of vitrimers, besides stress-relaxation, is their creep resistance. In contrast to thermoset materials, vitrimers tend to creep when heated above their $T_{g}$ due to the presence of exchangeable bonds, especially if these exchange reactions are fast. ${ }^{38}$ However, it is also known that the addition of irreversible crosslinks can greatly reduce the amount of irreversible deformation. ${ }^{51}$ Because those two characteristics, i.e. fast exchanges and irreversible linkages, are coexisting in the herein reported materials, their creep resistance has been characterized at different temperatures (Figure 6).
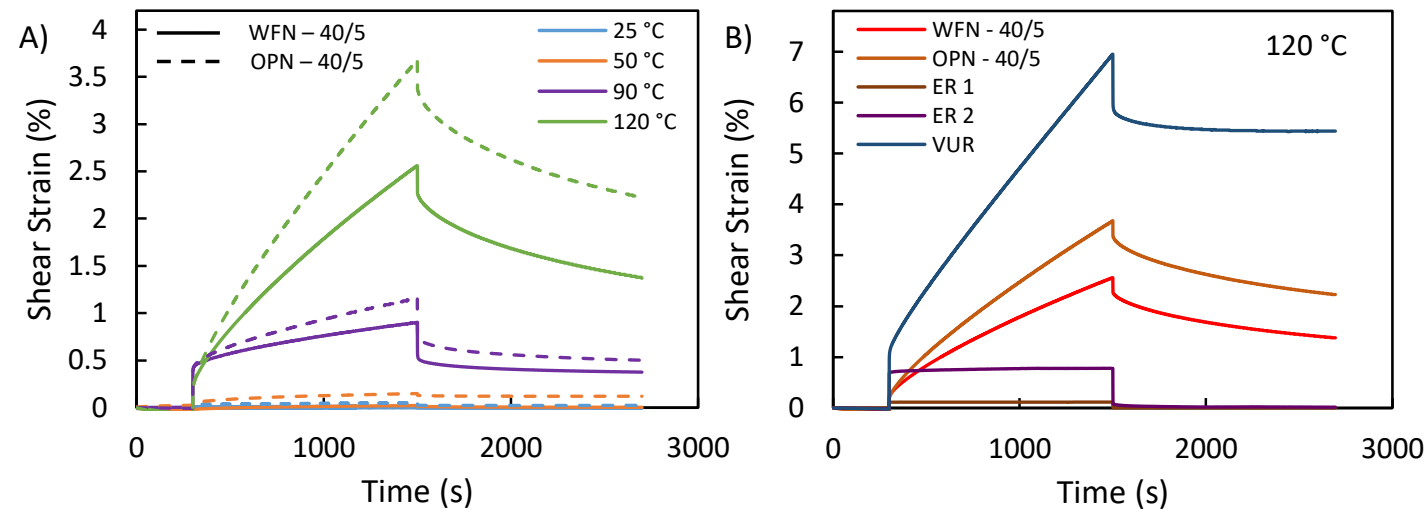

Figure 6. A) Creep recovery experiments of WFN (full line) and OPN (dashed line) materials at different temperatures with an applied stress of 5000 Pa for 20 minutes. B) Creep recovery experiments of $W F N_{40 / 5}, O P N_{40 / 5}, E R 1$, ER 2 and VUR materials at $120^{\circ} \mathrm{C}$ with an applied stress of 5000 Pa for 20 minutes.

On Figure 6A, which displays the creep recovery experiments of OPN and WFN-based vitrimers, it is clearly noticeable that below the $\mathrm{T}_{\mathrm{g}}$, no significant irreversible deformation is present in both materials. Nevertheless, when they are heated above their $\mathrm{T}_{\mathrm{g}}$, the amount of irreversible deformation, though still minimal $\left(<1 \%\right.$ at $\left.90{ }^{\circ} \mathrm{C}\right)$, starts to become more significant and increases with temperature $(>1 \%$ at $120^{\circ} \mathrm{C}$ ). Additionally, the higher irreversible deformation for the OPN-based material corroborates 
the faster exchange reaction observed in stress-relaxation experiments. However, as expected due to the lack of exchangeable bonds, the permanently crosslinked networks ER 1 and ER 2 still show a better creep resistance (Figure 6B and Figure S13-14). Nevertheless, $\mathrm{WFN}_{40 / 5}$ and $\mathrm{OPN}_{40 / 5}$ display a better creep resistance than the VUR (Figure 6B and Figure S12) despite having a faster relaxation, since a reduction of up to $75 \%$ in irreversible deformation was obtained at $120^{\circ} \mathrm{C}$. Therefore, these materials show the added value of using the vinylogous urethane chemistry to obtain epoxy vitrimers, since the hydroxyl groups in the polymer backbone lead to faster exchange reactions, while the irreversible nature of the epoxy linkages limits the creep at low temperature.

\section{Recyclability}

The materials' recyclability, which is a key aspect of vitrimers, was investigated by breaking the samples into small pieces and compression molding them in a hot press at $150^{\circ} \mathrm{C}$ for 15 minutes. After each recycling step, the $T_{g}$, the tensile properties and the FTIR spectra were verified (Table 1, Figure 7, Figure S16-20). Furthermore, the thermal degradation temperature (Figure S15-16), soluble fraction and the stress-relaxation (Figure S23-24) were also investigated and compared after the fourth and final recycling step (Table 1). 

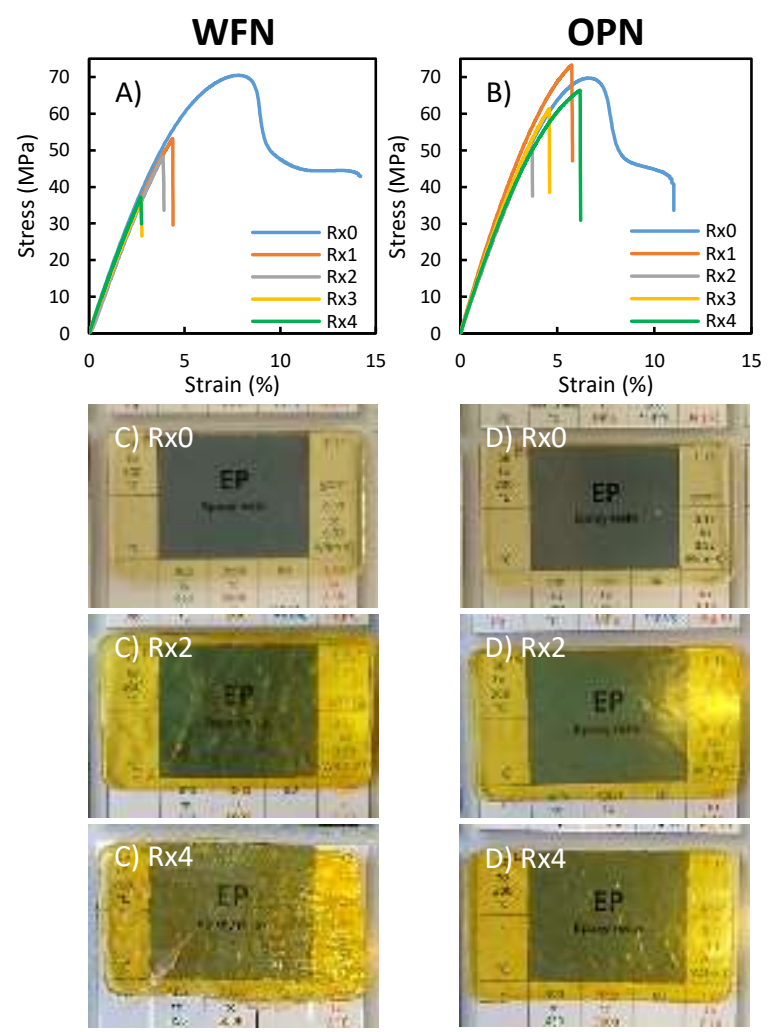

Figure 7. Recyclability studies for both WFN $W_{40 / 5}$ and $O P N_{40 / 5}$. A) and B) Stress-strain curves of $W F N_{40 / 5}$ and $O P N_{40 / 5}$ respectively after different recycling steps. C) and D) Visual aspect of the obtained samples after different recycling steps.

As already mentioned in Table 1 and as can be seen in Figure 7 A\&B, the apparent Young's modulus of both materials remained unchanged. However, the elongation property of these materials did change after the first recycling step, since necking suppression was observed in tensile strength experiments (Figure $7 \mathrm{~A}$ and B). Furthermore, a slight difference is also observable between the WFN- and OPN-based networks. As displayed in Figure 7A and Table 1, the elongation of $\mathrm{WFN}_{40 / 5}$ decreases over the course of the recycling steps, while for $\mathrm{OPN}_{40 / 5}$ only a clear elongation decrease was visible for the first recycling step (Figure 7B and Table 1). After the fourth and final recycling step, the influence of recycling on the stress-relaxation was also investigated. Here, the recycled sample of $\mathrm{WFN}_{40 / 5}$ had a slightly different behavior compared to the original sample (Figure S23). While the original sample could fully relax its stress (Figure 4), the recycled sample reached a plateau value, which resulted in at least $5 \%$ residual stress in the material. This could in principle be attributed to the oxidation of the pending amines, resulting in a lower amount of amines for the transamination reaction (and sample 
coloration). Indeed, the relaxation time also increased from $5.5 \mathrm{~s}$ to $6.5 \mathrm{~s}$ at $160^{\circ} \mathrm{C}$ for the four times recycled sample, corroborating this hypothesis. Another explanation is that, as a result of the recycling steps, some small domains appear that are unable to release the trapped stress, resulting in a percolated network as reported by Torkelson and co-workers ${ }^{51}$. The stress-relaxation measurements also display evidence for this second hypothesis, since roughly the same plateau value is reached at every investigated temperature (Figure S23), clearly indicating some irreversible domains in the network. On the other hand, the network obtained with the OPN approach did not display a plateau value and full stress relaxation could be obtained (Figure S24). This observation further supports the presence of a percolated network being the major reason for the plateau value obtained for the $\mathrm{WFN}_{40 / 5}$ sample. These conclusions are in good correlation with the results from the tensile tests, where $\mathrm{OPN}_{40 / 5}$ displayed full recyclability over the course of 4 recycling steps, while $\mathrm{WFN}_{40 / 5}$ displayed full recyclability up to 2 recycling steps. Furthermore, DSC measurements did not exhibit a significant difference over the course of the recycling process (Figure S17-18), since for both networks a $T_{g}$ of around $74{ }^{\circ} \mathrm{C}$ was maintained. In addition, no change was observed in the FTIR spectra of the recycled materials (Figure S19-20). Furthermore, after four recycling steps similar degradation temperatures and soluble fraction were obtained, compared to the original material (Table 1).

\section{Fiber reinforced polymer composite}

Fiber reinforced polymer composites (FRPC) are generally used in applications requiring high mechanical resistance such as in the aerospace industry. However, due to the use of irreversible crosslinked polymer networks, FRPC are impossible to reshape or recycle, leading to a lot of waste. In order to solve this problem, the use of vitrimers is receiving an increasing amount of attention. ${ }^{32,53,54}$ Since epoxy resins are often used as the polymer matrix of choice in composite materials, it was investigated whether the herein reported water-free procedure (Figure 22B) could be implemented to obtain composite materials with additional vitrimer properties. This would further show the wide 
applicability of the VU-containing curing agent by introducing recycling/reshaping features on top of the standard composite properties.

First, viscosity profiles were obtained for the mixture of EPIKOTE ${ }^{\mathrm{TM}}$ and the VU-containing curing agent at different temperatures (Figure S34-36) These profiles are necessary to determine what type of processing method could be used, since a low viscosity ( $<1000 \mathrm{mPa} . \mathrm{s})$ is necessary if resin transfer molding is envisioned. The profiles in Figure S34-36 showed that at elevated temperatures, the viscosity of the mixture could be reduced to values below $3000 \mathrm{mPa} . \mathrm{s}$ at $45^{\circ} \mathrm{C}$ and to around $600 \mathrm{mPa} . \mathrm{s}$ at $80^{\circ} \mathrm{C}$. However, these relatively low viscosities could only be maintained for a limited amount of time (few minutes) due to the reaction between the amines and the epoxy resin, resulting in a steep viscosity increase. Therefore, hand lamination was chosen as the preferred processing method (Figure 8A), since for this technique the control of viscosity is not a limiting factor. For this, both the EPIKOTE ${ }^{\mathrm{TM}}$ and the VU curing agent were heated to $45^{\circ} \mathrm{C}$, manually mixed and applied on the glass fiber fabric to obtain a one layer $\left(\left[0^{\circ}\right]_{1}\right)$ and a four layer composite $\left(\left[0^{\circ}\right]_{4}\right)$. The angle refers to the difference in fiber alignment between the layers.
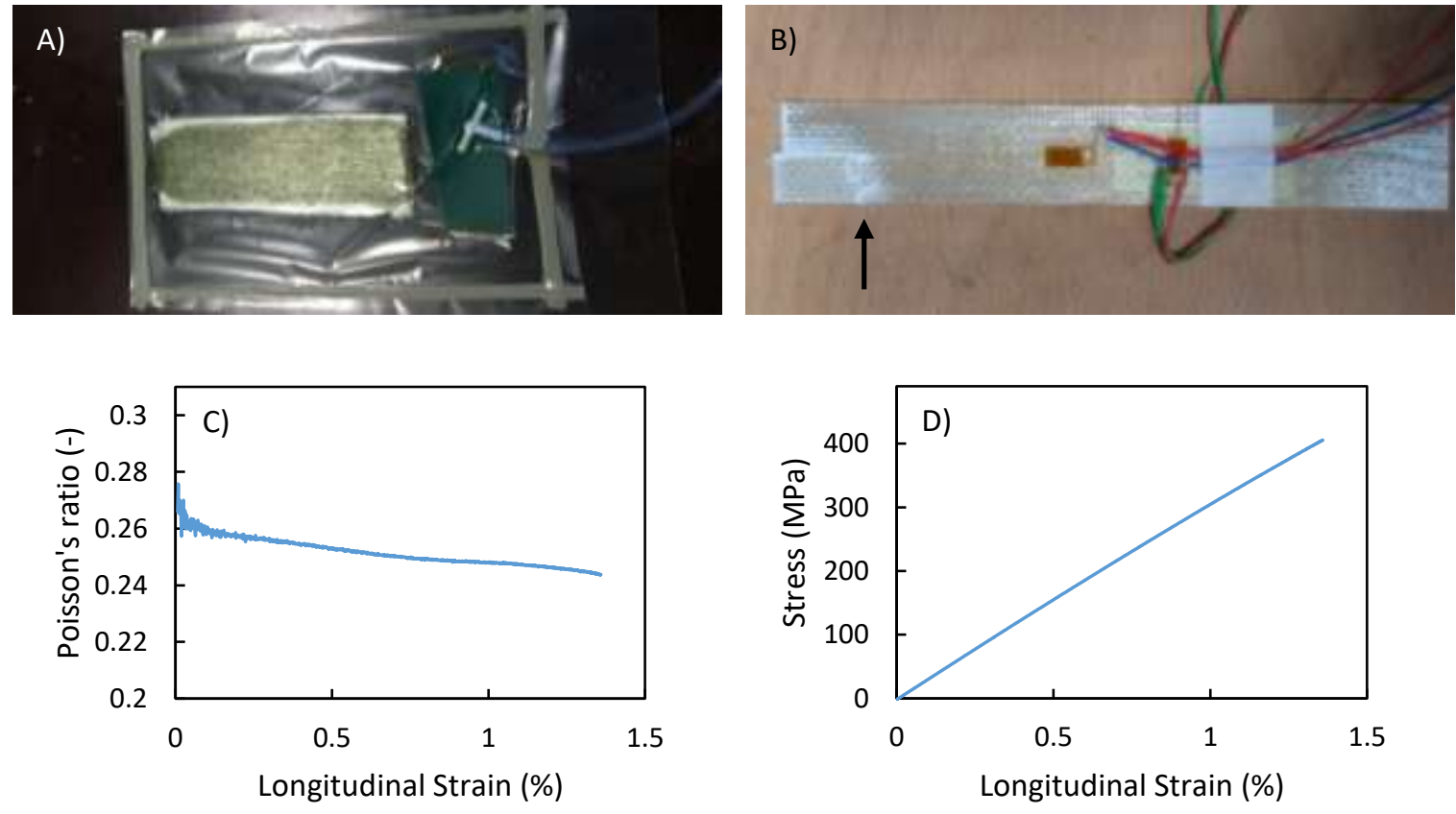
Figure 8. A) Example of an end-result of the hand lamination procedure. B) Four layer WFN-based composite after tensile test showing failure at the grip (indicated with a black arrow). C) Poisson's ratio as a function of strain. D) Stress-strain curve of the WFN-based composite.

Visually, the matrix displayed good adhesion to the fibers, however, some small porosities are still present, due to air trapped in the mixed formulation before it was applied to the fibers. The degassing step, necessary to prevent this phenomenon, could not be performed due to the fast viscosity increase. Despite these issues, the composite was further characterized starting with the fiber volume fraction of the four layered sample, which was determined based on TGA measurements (i.e. resin burning-off method). This resulted in a fiber volume fraction of $53 \%$ (Table S2), which is in agreement with the targeted $50 \%$. A tensile test was also performed on the four layered sample in the direction of the fibers $\left(0^{\circ}\right)$ and the values were compared to a unidirectional (UD) glass fabric reinforced epoxy reference (Figure 8D and Table 2). ${ }^{55}$ However, since the failure occurred at the grips (Figure 8B), the values obtained for the strain and elongation at failure are most likely underestimated.

Table 2. Mechanical properties of $W_{F} N_{40 / 5}$ composite.

\begin{tabular}{|c|c|c|}
\hline & $\begin{array}{c}\text { WFN }_{40 / 5} \text { composite } \\
{\left[0^{\circ}\right]_{4}}\end{array}$ & $\begin{array}{l}\text { UD glass fabric reinforced } \\
\text { epoxy }\left[0^{\circ}\right]_{8}\end{array}$ \\
\hline $\mathrm{E}_{11}(\mathrm{GPa})$ & 31.6 & 42 \\
\hline${ }^{a} X_{t}(\mathrm{MPa})$ & 405 & 939 \\
\hline${ }^{\mathrm{b}} \varepsilon_{11}(\%)$ & 1.36 & 2.5 \\
\hline${ }^{c} V_{12}$ & 0.259 & 0.259 \\
\hline
\end{tabular}

Additionally, no damage to the composite is visible, besides the fracture at the clamp, which is a good indication that the matrix adheres well to the fibers. Furthermore, the obtained stiffness $\left(E_{11}\right)$ is close to the expected value of a $50 \%$ glass fiber volume fraction containing composite. Moreover, the same 
Poisson's ratio was obtained as for the epoxy reference. Lastly, the reshapeability of the vinylogous urethane epoxy vitrimer composite was investigated using the one-layer composite (Figure 9).
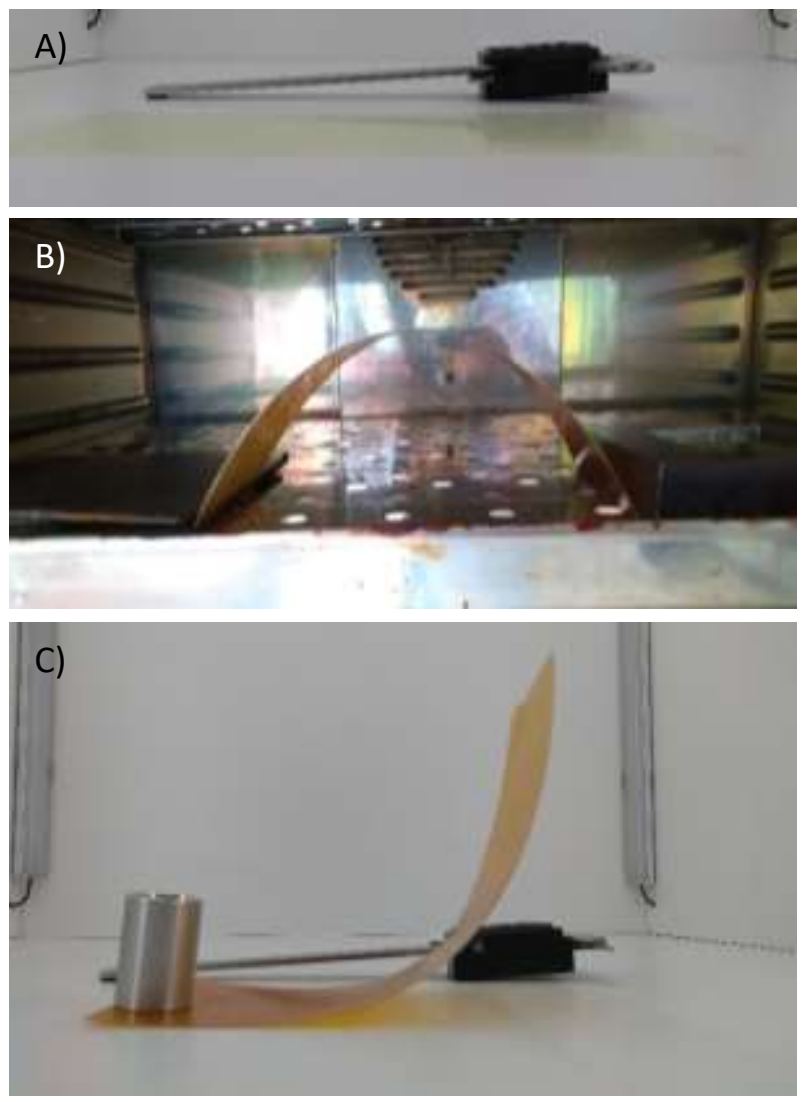

Figure 9. Reshaping of a vinylogous urethane epoxy vitrimer. A) Before reshaping. B) Reshaping set-up at $150{ }^{\circ} \mathrm{C}$ in an oven.

C) Result after reshaping.

The sample was placed in a pre-heated oven at $150^{\circ} \mathrm{C}$ for 10 minutes (together with the weights used for reshaping). After this, it was gradually deformed as shown in Figure 9B. Once the desired deformation was reached, the sample was kept in its new shape for 15 minutes to ensure full stress release due to reorganization of the vinylogous urethane matrix. Following this, the weights were removed, and the sample was cooled down. The sample maintained its new shape without visible damage to the matrix (Figure 9C and Figure S39F). This proof of concept study shows the possibilities of using the WFN procedure in composite production.

\section{Conclusion}


In summary, we have demonstrated that it is possible to obtain vinylogous urethane-containing epoxy/amine vitrimers via two facile procedures. On the one hand, a one-pot procedure with the release of water in the resin matrix was developed, and on the other hand a water-free two-step procedure (WFN) was developed wherein epoxy vitrimers can be cured without the release of water or other volatiles. In the WFN method, water is removed in a prepolymerisation step, leading to an oligomeric polyfunctional amine curing agent, also containing dynamic VU moieties, enabling a drop-in solution for classical epoxy/amine formulations.

Additionally, the two procedures result in nearly identical materials and both display very fast stress-relaxation with relaxation times as low as $3.5 \mathrm{~s}$ at $160^{\circ} \mathrm{C}$. This fast exchange, even in comparison to polymer networks wherein the VU-moieties are present in every repeating unit, can be rationalized by the presence of pending hydroxyl groups, which have a demonstrated accelerating effect on the transamination reaction of vinylogous urethanes. These highly dynamic, catalyst free materials can be easily processed and recycled two to four times while maintaining their properties. The material properties are also similar to the commercial VU-free epoxy reference material. Lastly, we demonstrated that not only the resins but also the corresponding glass-fiber reinforced composites can be reshaped.

\section{Associated content}

Supporting Information. Supplemental figures, tables and supplemental pictures, additional experiments and material characterization.

\section{Notes}

The authors declare no competing financial interest.

\section{Acknowledgements}

Y.S. acknowledges the Research Foundation-Flanders (FWO) for funding of his Ph.D. fellowship. Bernhard De Meyer and Bastiaan Dhanis are gratefully acknowledged for technical and synthetic 
support. Dr. Nezha Badi and Christian Taplan are thanked for valuable input. F.D.P and J.W.

acknowledge UGent (BOF-GOA) and VLAIO-funding (Moonshot program, Catalisti).

\section{References}

1. Petrović, Z. S.; Zlatanić, A.; Lava, C. C.; Sinadinović-Fišer, S. Epoxidation of soybean oil in toluene with peroxoacetic and peroxoformic acids - kinetics and side reactions. European Journal of Lipid Science and Technology 2002, 104 (5), 293-299 DOI: 10.1002/1438-

9312(200205)104:5<293::aid-ejlt293>3.0.co;2-w.

2. Derouet, D.; Brosse, J.-C.; Challioui, A. Alcoholysis of epoxidized polyisoprenes by direct opening of oxirane rings with alcohol derivatives 2. Study on epoxidized 1,4-polyisoprene. Eur. Polym. J. 2001, 37 (7), 1327-1337 DOI: http://dx.doi.org/10.1016/S0014-3057(00)00267-6.

3. Challioui, A.; Derouet, D.; Oulmidi, A.; Brosse, J. C. Alkoxylation of epoxidized polyisoprene by opening of oxirane rings with alcohols. Polym. Int. 2004, 53 (8), 1052-1059 DOI: 10.1002/pi.1486.

4. Zhao, Y. X.; Huang, B. C.; Yao, W.; Cong, H. L.; Shao, H. F.; Du, A. H. Epoxidation of high trans1,4-polyisoprene and its properties. J. Appl. Polym. Sci. 2008, 107 (5), 2986-2993 DOI:

10.1002/app.27394.

5. Jin, F.-L.; Li, X.; Park, S.-J. Synthesis and application of epoxy resins: A review. Journal of Industrial and Engineering Chemistry 2015, 29, 1-11 DOI: https://doi.org/10.1016/j.jiec.2015.03.026.

6. Rafique, I.; Kausar, A.; Anwar, Z.; Muhammad, B. Exploration of Epoxy Resins, Hardening Systems, and Epoxy/Carbon Nanotube Composite Designed for High Performance Materials: A Review. Polymer-Plastics Technology and Engineering 2016, 55 (3), 312-333 DOI: 10.1080/03602559.2015.1070874.

7. Hoyle, C. E.; Lowe, A. B.; Bowman, C. N. Thiol-click chemistry: a multifaceted toolbox for small molecule and polymer synthesis. Chem. Soc. Rev. 2010, 39 (4), 1355-1387 DOI: 10.1039/B901979K.

8. Dušek, K.; Bleha, M.; Luňák, S. Curing of epoxide resins: Model reactions of curing with amines. Journal of Polymer Science: Polymer Chemistry Edition 1977, 15 (10), 2393-2400 DOI: doi:10.1002/pol.1977.170151009.

9. Charlesworth, J. An analysis of the substitution effects involved in diepoxide-diamine copolymerization reactions. Journal of Polymer Science: Polymer Chemistry Edition 1980, 18 (2), 621628 DOI: doi:10.1002/pol.1980.170180221.

10. Montarnal, D.; Capelot, M.; Tournilhac, F.; Leibler, L. Silica-Like Malleable Materials from Permanent Organic Networks. Science 2011, 334 (6058), 965-968 DOI: 10.1126/science.1212648.

11. Weiss, H. K. Anhydride curing agents for epoxy resins. Industrial and Engineering Chemistry 1957, 49 (7), 1089-1090 DOI: 10.1021/ie50571a024.

12. Kloxin, C. J.; Scott, T. F.; Adzima, B. J.; Bowman, C. N. Covalent Adaptable Networks (CANs): A Unique Paradigm in Cross-Linked Polymers. Macromolecules 2010, 43 (6), 2643-2653 DOI: 10.1021/ma902596s.

13. Winne, J. M.; Leibler, L.; Du Prez, F. E. Dynamic covalent chemistry in polymer networks: a mechanistic perspective. Polym. Chem. 2019, 10, 6091-6108 DOI: 10.1039/C9PY01260E.

14. Legrand, A.; Soulie-Ziakovic, C. Silica-Epoxy Vitrimer Nanocomposites. Macromolecules 2016, 49 (16), 5893-5902 DOI: 10.1021/acs.macromol.6b00826.

15. Demongeot, A.; Groote, R.; Goossens, H.; Hoeks, T.; Tournilhac, F.; Leibler, L. Cross-Linking of Poly(butylene terephthalate) by Reactive Extrusion Using Zn(II) Epoxy-Vitrimer Chemistry.

Macromolecules 2017, 50 (16), 6117-6127 DOI: 10.1021/acs.macromol.7b01141.

16. Zhang, H.; Cai, C.; Liu, W.; Li, D.; Zhang, J.; Zhao, N.; Xu, J. Recyclable Polydimethylsiloxane Network Crosslinked by Dynamic Transesterification Reaction. Sci. Rep. 2017, 7, 11833 DOI:

10.1038/s41598-017-11485-6. 
17. Chen, J. H.; An, X. P.; Li, Y. D.; Wang, M.; Zeng, J. B. Reprocessible Epoxy Networks with Tunable Physical Properties: Synthesis, Stress Relaxation and Recyclability. Chin. J. Polym. Sci. 2018, 36 (5), 641-648 DOI: 10.1007/s10118-018-2027-9.

18. Liu, T.; Hao, C.; Zhang, S.; Yang, X.; Wang, L.; Han, J.; Li, Y.; Xin, J.; Zhang, J. A Self-Healable High Glass Transition Temperature Bioepoxy Material Based on Vitrimer Chemistry. Macromolecules 2018, DOI: 10.1021 acs.macromol.8b01010.

19. Capelot, M.; Unterlass, M. M.; Tournilhac, F.; Leibler, L. Catalytic Control of the Vitrimer Glass Transition. ACS Macro Lett. 2012, 1 (7), 789-792 DOI: 10.1021/mz300239f.

20. Shi, Q.; Yu, K.; Kuang, X.; Mu, X.; Dunn, C. K.; Dunn, M. L.; Wang, T.; Jerry Qi, H. Recyclable 3D printing of vitrimer epoxy. Mater. Horizons 2017, 4 (4), 598-607 DOI: 10.1039/C7MH00043J.

21. Denissen, W.; Rivero, G.; Nicolay, R.; Leibler, L.; Winne, J. M.; Du Prez, F. E. Vinylogous Urethane Vitrimers. Adv. Funct. Mater. 2015, 25 (16), 2451-2457 DOI: 10.1002/adfm.201404553.

22. Stukenbroeker, T.; Wang, W. D.; Winne, J. M.; Du Prez, F. E.; Nicolay, R.; Leibler, L. Polydimethylsiloxane quenchable vitrimers. Polym. Chem. 2017, 8 (43), 6590-6593 DOI: 10.1039/c7py01488k.

23. Denissen, W.; Droesbeke, M.; Nicolaÿ, R.; Leibler, L.; Winne, J. M.; Du Prez, F. E. Chemical control of the viscoelastic properties of vinylogous urethane vitrimers. Nat. Commun. 2017, 8, 14857 DOI: $10.1038 /$ ncomms14857.

24. Liu, Z.; Zhang, C.; Shi, Z.; Yin, J.; Tian, M. Tailoring vinylogous urethane chemistry for the cross-linked polybutadiene: Wide freedom design, multiple recycling methods, good shape memory behavior. Polymer 2018, 148, 202-210 DOI: https://doi.org/10.1016/j.polymer.2018.06.042.

25. Taynton, P.; Zhu, C.; Loob, S.; Shoemaker, R.; Pritchard, J.; Jin, Y.; Zhang, W. Re-healable polyimine thermosets: polymer composition and moisture sensitivity. Polym. Chem. 2016, 7 (46), 7052-7056 DOI: 10.1039/C6PY01395C.

26. Zhang, H.; Wang, D.; Liu, W.; Li, P.; Liu, J.; Liu, C.; Zhang, J.; Zhao, N.; Xu, J. Recyclable polybutadiene elastomer based on dynamic imine bond. J. Polym. Sci. Part A: Polym. Chem. 2017, 55, 2011-2018 DOI: 10.1002/pola.28577.

27. Lei, X.; Jin, Y.; Sun, H.; Zhang, W. Rehealable imide-imine hybrid polymers with full recyclability. Journal of Materials Chemistry A 2017, 5 (40), 21140-21145 DOI: 10.1039/C7TA07076D. 28. Liu, H.; Zhang, H.; Wang, H.; Huang, X.; Huang, G.; Wu, J. Weldable, malleable and programmable epoxy vitrimers with high mechanical properties and water insensitivity. Chemical Engineering Journal 2019, 368, 61-70 DOI: https://doi.org/10.1016/j.cej.2019.02.177.

29. Spiesschaert, Y.; Guerre, M.; Imbernon, L.; Winne, J. M.; Du Prez, F. Filler reinforced polydimethylsiloxane-based vitrimers. Polymer 2019, 172, 239-246 DOI: https://doi.org/10.1016/j.polymer.2019.03.075.

30. Zhao, S.; Abu-Omar, M. M. Recyclable and Malleable Epoxy Thermoset Bearing Aromatic Imine Bonds. Macromolecules 2018, 51 (23), 9816-9824 DOI: 10.1021/acs.macromol.8b01976.

31. Lv, C.; Zhao, K.; Zheng, J. A Highly Stretchable Self-Healing Poly(dimethylsiloxane) Elastomer with Reprocessability and Degradability. Macromol. Rapid Commun. 2018, 39 (8), 1700686 DOI: 10.1002/marc.201700686.

32. Ruiz de Luzuriaga, A.; Martin, R.; Markaide, N.; Rekondo, A.; Cabañero, G.; Rodríguez, J.; Odriozola, I. Epoxy resin with exchangeable disulfide crosslinks to obtain reprocessable, repairable and recyclable fiber-reinforced thermoset composites. Mater. Horizons 2016, 3 (3), 241-247 DOI: 10.1039/C6MH00029K.

33. Imbernon, L.; Oikonomou, E. K.; Norvez, S.; Leibler, L. Chemically crosslinked yet reprocessable epoxidized natural rubber via thermo-activated disulfide rearrangements. Polym. Chem. 2015, 6 (23), 4271-4278 DOI: 10.1039/C5PY00459D.

34. Nishimura, Y.; Chung, J.; Muradyan, H.; Guan, Z. B. Silyl Ether as a Robust and Thermally Stable Dynamic Covalent Motif for Malleable Polymer Design. J. Am. Chem. Soc. 2017, 139 (42), 14881-14884 DOI: 10.1021/jacs.7b08826. 
35. Tretbar, C. A.; Neal, J. A.; Guan, Z. B. Direct Silyl Ether Metathesis for Vitrimers with Exceptional Thermal Stability. J. Am. Chem. Soc. 2019, 141 (42), 16595-16599 DOI: 10.1021/jacs.9b08876.

36. Fortman, D. J.; Brutman, J. P.; Cramer, C. J.; Hillmyer, M. A.; Dichtel, W. R. Mechanically Activated, Catalyst-Free Polyhydroxyurethane Vitrimers. J. Am. Chem. Soc. 2015, 137 (44), 1401914022 DOI: 10.1021/jacs.5b08084.

37. Snyder, R. L.; Fortman, D. J.; De Hoe, G. X.; Hillmyer, M. A.; Dichtel, W. R. Reprocessable AcidDegradable Polycarbonate Vitrimers. Macromolecules 2018, 51 (2), 389-397 DOI: 10.1021/acs.macromol.7b02299.

38. Denissen, W.; Winne, J. M.; Du Prez, F. E. Vitrimers: permanent organic networks with glasslike fluidity. Chem. Sci. 2016, 7 (1), 30-38 DOI: 10.1039/c5sc02223a.

39. Obadia, M. M.; Jourdain, A.; Cassagnau, P.; Montarnal, D.; Drockenmuller, E. Tuning the Viscosity Profile of lonic Vitrimers Incorporating 1,2,3-Triazolium Cross-Links. Adv. Funct. Mater. 2017, 27 (45), 1703258 DOI: 10.1002/adfm.201703258.

40. Röttger, M.; Domenech, T.; van der Weegen, R.; Breuillac, A.; Nicolaÿ, R.; Leibler, L. Highperformance vitrimers from commodity thermoplastics through dioxaborolane metathesis. Science 2017, 356 (6333), 62-65 DOI: 10.1126/science.aah5281.

41. Ishibashi, J. S. A.; Kalow, J. A. Vitrimeric Silicone Elastomers Enabled by Dynamic Meldrum's Acid-Derived Cross-Links. ACS Macro Lett. 2018, 7 (4), 482-486 DOI: 10.1021/acsmacrolett.8b00166.

42. Su, W.-L.; Liu, Y.-L. Self-crosslinkable and modifiable polysiloxanes possessing Meldrum's acid groups. Polym. Chem. 2018, DOI: 10.1039/C8PY01173G.

43. Mo, R. B.; Hu, J.; Huang, H. W.; Sheng, X. X.; Zhang, X. Y. Tunable, self-healing and corrosion inhibiting dynamic epoxy-polyimine network built by post-crosslinking. Journal of Materials Chemistry A 2019, 7 (7), 3031-3038 DOI: 10.1039/c8ta11546j.

44. Mai, V.-D.; Shin, S.-R.; Lee, D.-S.; Kang, I. Thermal Healing, Reshaping and Ecofriendly Recycling of Epoxy Resin Crosslinked with Schiff Base of Vanillin and Hexane-1,6-Diamine. Polymers 2019, 11 (2), 293.

45. Wu, X.; Yang, X.; Yu, R.; Zhao, X.-J.; Zhang, Y.; Huang, W. A facile access to stiff epoxy vitrimers with excellent mechanical properties via siloxane equilibration. J. Mater. Chem. A 2018, 6 (22), 10184-10188 DOI: 10.1039/C8TA02102C.

46. Matxain, J. M.; Asua, J. M.; Ruipérez, F. Design of new disulfide-based organic compounds for the improvement of self-healing materials. Physical Chemistry Chemical Physics 2016, 18 (3), 17581770 DOI: 10.1039/C5CP06660C.

47. Scott, T. F.; Schneider, A. D.; Cook, W. D.; Bowman, C. N. Photoinduced plasticity in crosslinked polymers. Science 2005, 308 (5728), 1615-1617 DOI: 10.1126/science.1110505.

48. Guerre, M.; Taplan, C.; Nicolay, R.; Winne, J. M.; Du Prez, F. E. Fluorinated vitrimer elastomers with a dual temperature response. J. Am. Chem. Soc. 2018, 13272-13284 DOI: 10.1021/jacs.8b07094.

49. Danisman, M.; Tuncol, G.; Kaynar, A.; Sozer, E. M. Monitoring of resin flow in the resin transfer molding (RTM) process using point-voltage sensors. Composites Science and Technology 2007, 67 (3), 367-379 DOI: https://doi.org/10.1016/j.compscitech.2006.09.011.

50. Schuster, J.; Govignon, Q.; Bickerton, S., Processability of Biobased Thermoset Resins and Flax Fibres Reinforcements Using Vacuum Assisted Resin Transfer Moulding. 2014; Vol. 4, p 1-11.

51. Li, L.; Chen, X.; Jin, K.; Torkelson, J. M. Vitrimers Designed Both To Strongly Suppress Creep and To Recover Original Cross-Link Density after Reprocessing: Quantitative Theory and Experiments. Macromolecules 2018, 51 (15), 5537-5546 DOI: 10.1021/acs.macromol.8b00922.

52. Delahaye, M.; Winne, J. M.; Du Prez, F. E. Internal Catalysis in Covalent Adaptable Networks: Phthalate Monoester Transesterification As a Versatile Dynamic Cross-Linking Chemistry. J. Am.

Chem. Soc. 2019, 141 (38), 15277-15287 DOI: 10.1021/jacs.9b07269.

53. Chabert, E.; Vial, J.; Cauchois, J.-P.; Mihaluta, M.; Tournilhac, F. Multiple welding of long fiber epoxy vitrimer composites. Soft Matter 2016, 12 (21), 4838-4845 DOI: 10.1039/C6SM00257A. 
54. Taynton, P.; Ni, H.; Zhu, C.; Yu, K.; Loob, S.; Jin, Y.; Qi, H. J.; Zhang, W. Repairable Woven Carbon Fiber Composites with Full Recyclability Enabled by Malleable Polyimine Networks. Adv. Mater. 2016, 28 (15), 2904-2909 DOI: 10.1002/adma.201505245.

55. Denissen, W.; De Baere, I.; Van Paepegem, W.; Leibler, L.; Winne, J.; Du Prez, F. E. Vinylogous Urea Vitrimers and Their Application in Fiber Reinforced Composites. Macromolecules 2018, 51 (5), 2054-2064 DOI: 10.1021/acs.macromol.7b02407. 